\title{
Salidroside could enhance the cytotoxic effect of L-OHP on colorectal cancer cells
}

\author{
XIAOMING SHI, WEI ZHAO, YONGBIN YANG, SHENGCHUN WU and BONAN LV \\ Department of General Surgery, Hebei General Hospital, Shijiazhuang, Hebei 050051, P.R. China
}

Received February 16, 2017; Accepted September 22, 2017

DOI: $10.3892 / \mathrm{mmr} .2017 .7846$

\begin{abstract}
Evidence has suggested that salidroside inhibits the proliferation and invasion of renal clear cell, lung, breast, and colon cancer. However, effect of salidroside on colorectal cancer (CRC) cells against oxaliplatin (L-OHP) resistance remains unclear. In the present study, the CRC HT-29 cell line and L-OHP resistance HT-29/L-OHP cell line were used to evaluate the effect, and mechanism of salidroside on L-OHP resistance. The results demonstrated that the activity of HT-29 cells was lower compared with that of HT-29/L-OHP cells following L-OHP intervention, and was accompanied with varied expression levels of drug resistant proteins. The combination of salidroside and L-OHP weakened cell activity significantly compared single utilization. Compared with the control group, salidroside intervention resulted in a higher percentage of HT-29/L-OHP cells in the $\mathrm{G}_{0} / \mathrm{G}_{1}$ stage, and reduced percentage in the $\mathrm{G}_{2} / \mathrm{M}$ stage, but no significant variation in the S stage. The HT-29/L-OHP cells exhibited increased apoptosis rates and caspase-3 activity, but decreased metastatic, and invasive abilities following salidroside intervention. Quantitative polymerase chain reaction and western blot analysis detected variations in the expression levels of associated genes in HT-29/L-OHP cells following salidroside intervention. In all, the results of the present study revealed that salidroside is able to decrease the activity and invasive capacity of HT-29/L-OHP cells, and treatment with salidroside is associated with increased apoptosis of cancer cells through the regulation of certain genes.
\end{abstract}

\section{Introduction}

Colorectal cancer (CRC) has become a fatal threat with the 4th highest morbidity and 5th highest mortality in all cancers (1), and incidence of CRC also increased dramatically

Correspondence to: Professor Xiaoming Shi, Department of General Surgery, Hebei General Hospital, 348 Heping Western Road, Shijiazhuang, Hebei 050051, P.R. China

E-mail: shixiaoming1999@126.com

Key words: colorectal cancer, salidroside, drug resistance, L-OHP, chemosensitivity in vitro in China $(2,3)$. Due to difficulty of detecting this disease at early stage, the majority of patients were diagnosed as being at the progressive stage at the first visit to hospital. Although the chemotherapy, one of the main treatments for CRC, is being updated, the outcome remains unsatisfactory and results in poor prognosis (4-10). Cancer cells can develop resistance to chemotherapeutic drugs, such as oxaliplatin (L-OHP), and this has been demonstrated as the crucial factor of chemotherapy failure (11-13). Given this fact, reversing the drug resistance of cancer cells has been regarded as a practical method for enhancing chemotherapy efficacy and improving the prognosis of patients.

Salidroside is the active ingredient of Rhodiolae, a plant has been used in the Far East and Russia traditional medicine for a variety of diseases (14-16). Evidence has demonstrated that salidroside can inhibit the proliferation and invasion of abnormal cells in renal clear cell cancer (17), lung cancer (18), breast cancer (19), and colon cancer (20). However, the effect of salidroside on CRC cells against L-OHP resistance remains unclear.

In present study, we assessed the effect of salidroside on the activity, cycle and apoptosis of L-OHP resistance CRC cells in vitro. We also detected the expression variation of drug-resistant genes before and after salidroside intervention, in terms of MRP-1, P-gp, LOXL2, Survivin, Livin, Bcl-2 and Bax, and also investigated the molecular mechanism and potential clinical value of salidroside.

\section{Materials and methods}

Cell lines and cell culture. CRC cell line HT-29 was obtained from the Institute of Biochemistry and Cell Biology, Shanghai Institutes for Biological Sciences, Chinese Academy of Sciences (Shanghai, China). L-OHP-resistant CRC line HT-29/L-OHP was cultivated and identified by our research team using increased concentration, which reached a resistant index (RI) of 4.26 for L-OHP. HT-29 and HT-29/L-OHP were cultivated under $5 \% \mathrm{CO}_{2}$ and $37^{\circ} \mathrm{C}$ with Dulbecco's modified Eagle's medium (DMEM) (Gibco; Thermo Fisher Scientific, Inc., Waltham, MA, USA), which contains $10 \%$ fetal calf serum (FCS). Trypsin (0.25\%) (Gibco; Thermo Fisher Scientific, Inc.) with $0.02 \%$ EDTA was used for the digestive transfer. L-OHP $(1.5 \mu \mathrm{g} / \mathrm{ml})$ was added into the substrate of HT-29/L-OHP to maintain the drug-resistant phenotype until 2 weeks before the experiment. 
Table I. Primer sequences for quantitative real-time PCR.

\begin{tabular}{lll}
\hline Genes & \multicolumn{1}{c}{ Forward primer $\left(5^{\prime}-3^{\prime}\right)$} & \multicolumn{1}{c}{ Reverse primer (5'-3') } \\
\hline MRP-1 & CATCAGCAGGCACCACAAC & TTCCAGGTCTCCTCCTTCTTG \\
MDR1 & GAATGTTCAGTGGCTCCGAG & ACAATCTCTTCCTGTGACACC \\
LOXL2 & CACCCACTATGACCTGCTGA & TCTTCTGGATGTCTCCTTCACA \\
Survivin & GCCAGATTTGAATCGCGGGA & GCAGTGGATGAAGCCAGCCT \\
Livin & TCCACAGTGTGCAGGAGACT & ACGGCACAAAGACGATGGAC \\
Bcl-2 & TGTGTGGAGAGCGTCAACC & TGGATCCAGGTGTGCAGGT \\
Bax & TTTCTGACGGCAACTTCAAC & AGTCCAATGTCCAGCCCAT \\
GAPDH & GACCCCTTCATTGACCTCAAC & CGCTCCTGGAAGATGGTGAT
\end{tabular}

Detection of cell activity with SRB assay. Single-cell suspension was prepared and adjusted to $10^{6} / \mathrm{ml}$ whereas salidroside (Sigma-Aldrich; Merck KGaA, Darmstadt, Germany) and L-OHP (Laboratoires Thissen, Belgium) were added after the cell attachment. Cell fixation was conducted using 50\% TCA $(50 \mu \mathrm{l})$ under $4^{\circ} \mathrm{C}$ for $1 \mathrm{~h}$ which was followed by elution (5 times) using ultrapure water and air drying. $100 \mu \mathrm{l}$ sulphorhodamine B (SRB; Pierce, Rockford, IL, USA) were added to each well for $10 \mathrm{~min}$ in dark place $\left(25^{\circ} \mathrm{C}\right)$ and then discarded. The optical density (OD) value was detected using microplate reader under $545 \mathrm{~nm}$. The cell activity was presented as the OD ratio which is defined as OD ratio in experimental group/OD ration in control groups. This experiment was repeated for 3 times.

Detection of the expressions of targeted proteins. After quantification (ABC assay), $60 \mu \mathrm{g}$ proteins from specimens in each group was purified by polyacrylamide gel electrophoresis (PAGE) and then transferred to PVDF membrane for overnight $\left(4^{\circ} \mathrm{C}\right)$ after adding first antibody or $\beta$-actin (Sigma-Aldrich; Merck KGaA). Elution was performed using TBST for 3 times and then the second antibody marked by horseradish peroxidase (HRP) was added. After incubation under $25^{\circ} \mathrm{C}$ for $1 \mathrm{~h}$, chemiluminescence was applied for coloration following by stripe scanning.

Detection of cell cycle. After centrifugation, cells were fixed using $70 \%$ alcohol under $4^{\circ} \mathrm{C}$. The cell cycle was determined by flow cytometry (FCM; Becton-Dickinson, San Jose, CA, USA) with $100 \mu \mathrm{l}$ cell suspension $\left(1 \times 10^{6} / \mathrm{ml}\right)$ which was pretreated by PBS (containing $50 \mu \mathrm{g} / \mathrm{ml}$ propidium iodide, $100 \mu \mathrm{g} / \mathrm{ml}$ RNase A and $0.2 \%$ Triton X-100) under $4^{\circ} \mathrm{C}$ for 30 min in dark.

Detection of cell apoptosis. The cell apoptosis rate was detected by Annexin V-FITC/propidium iodide (PI) detection kit (Jiamei North Biological Technology Co., Ltd., Beijing, China) with $100 \mu \mathrm{l}$ cell suspension $\left(1 \times 10^{6} / \mathrm{ml}\right)$ which was pretreated using $5 \mu \mathrm{l}$ Annexin V-FITC and $10 \mu \mathrm{l}$ PI under $25^{\circ} \mathrm{C}$ for 15 min in dark.

Cell scratch assay. Single-cell suspension was adjusted to $5 \times 10^{4} / \mathrm{ml}$ and then transferred to the 24 -well plate for cultivating during a period of $48 \mathrm{~h}$. After discarding DMEM, scratching was conducted using the $200 \mu \mathrm{l}$ tips which was followed by 24-h culture using serum-free medium. Wound healing was inspected under microscope after washing out the scratched cells. The distance and ratio of cell migration were calculated.

Transwell chamber assay. The pore size of the polycarbonate membrane of the Transwell chamber is $8 \mu \mathrm{m}$ where the upper chamber was membraned using $100 \mu \mathrm{l}$ Matrigel under ultraviolet radiation for $2 \mathrm{~h}$. Cells were inoculated to the 6 -well plate for $1 \times 10^{6} / \mathrm{ml}$ per well and intervened for $24 \mathrm{~h}$ when growing up to $60-70 \%$. Single-cell suspension $(200 \mu 1)$ was inoculated to the upper chamber and then placed to the 24-well plate, while the lower chamber was cultivated using DMEM (containing 10\% FCS). After $24 \mathrm{~h}$ culture, the Matrigel and cells in the upper chamber were removed using aseptic swab. The polycarbonate membrane was fixed using methanol for $10 \mathrm{~min}$ and then colored by crystal violet. Cell counts were calculated by inspecting 5 random fields under high magnification. The inhibition rate of invasion was defined as (1 - counts of invasive cells in experimental group/counts of invasive cells in control group) $x 100 \%$. The procedure was repeated for 3 times.

Real-time PCR assay. Total RNA of cells were extracted and retransformed into cDNA, which was then amplified by PCR for detecting the mRNA expressions of targeted molecules. The primers are shown in Table I. The amplified results were identified using agarose gel electrophoresis. The results of fluorogenic quantitative PCR was calculated using $2^{-\Delta \Delta C t}$ method with $\beta$-actin working as internal reference.

Detection of caspase-3 activity. N-acetyl-Asp-Glu-Val-As p-7-amino-4-trifluorom ethylcoumarin (Ac-DEVD-AFC; Calbiochem, San Diego, CA, USA) was utilized to detect the activity of caspase-3, caspase-3 analysis buffer $(50 \mathrm{mM}$ HEPES, pH 7.4, $100 \mathrm{mM} \mathrm{NaCl,} 1 \mathrm{mM}$ EDTA and $10 \mathrm{mM}$ DTT) was added into $15 \mu \mathrm{l}$ protein extracts. After incubation under $37^{\circ} \mathrm{C}$ for $2 \mathrm{~h}$, specimens were treated using $15 \mu \mathrm{l}$ Ac-DEVD-AFC solution ( $2 \mathrm{mM}$ ) and then detected by ELIASA reader under $505 \mathrm{~nm}$.

Statistical analysis. Mean \pm SD was recorded to demonstrate the results of cell activity, western blot analysis, cell cycle, apoptosis, invasion/metastasis, values of fluorogenic quantitative PCR test and caspase- 3 activity. The heterogeneities 


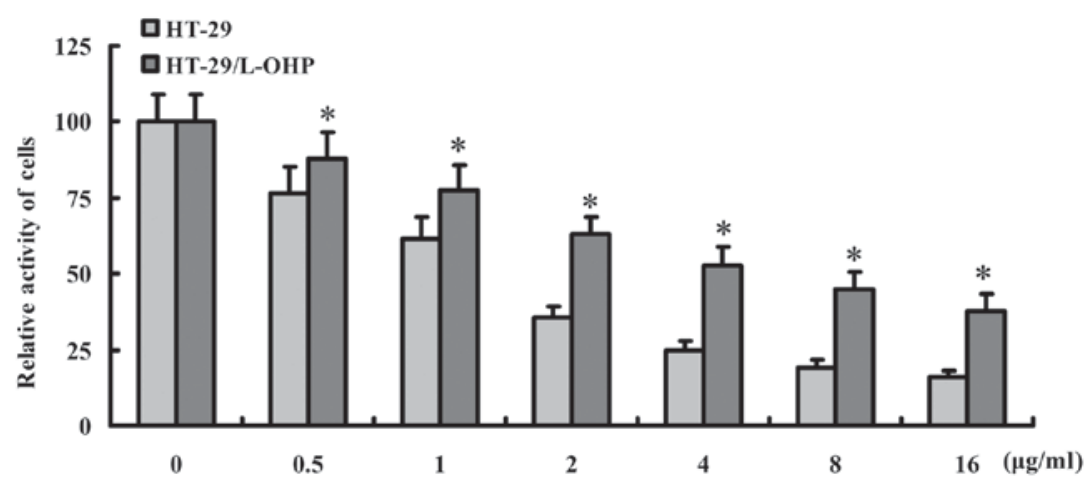

Figure 1. Sensitivity of HT-29 and HT-29/L-OHP cells to L-OHP. HT-29 and HT-29/L-OHP cells were treatment with different dosages of L-OHP for 48 h, and the activity of cells were illustrated as Fig. 1, which showed that activity of HT-29 was less obvious than HT-29/L-OHP after the intervention (P<0.05). "P $<0.05$ vs. HT- 29 cells.
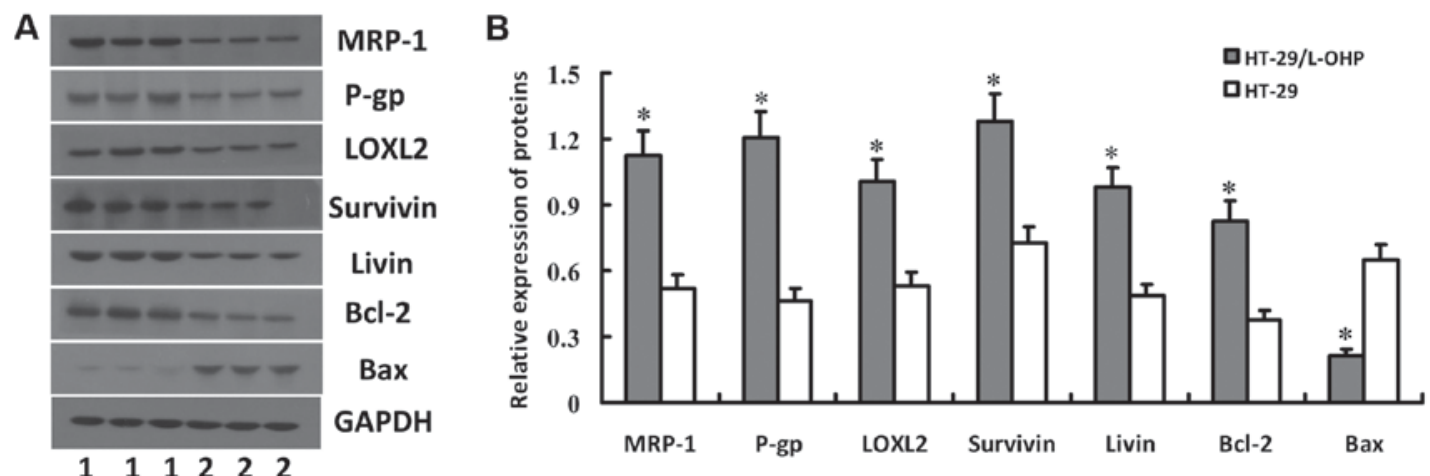

Figure 2. Expression of MRP-1, P-gp, LOXL2, Survivin, Livin, Bcl-2 and Bax proteins in HT-29 and HT-29/L-OHP cells. HT-29 and HT-29/L-OHP cells were subjected to western blot analysis to detect the expression of MRP-1, P-gp, LOXL2, Survivin, Livin, Bcl-2 and Bax proteins. Gel electrophoresis map was shown in (A) and relative protein levels were shown in (B). "P<0.05 vs. HT-29 cells.

between different groups were detected using one-way ANOVA and Dunnett's test by SPSS 19.0 (SPSS, Inc., Chicago, IL, USA). $\mathrm{P}<0.05$ was considered to indicate a statistically significant difference.

\section{Results}

The effect of L-OHP on HT-29 and HT-29/L-OHP cells. To evaluate the effect of L-OHP on HT-29 and HT-29/L-OHP cells, different dosages of L-OHP were applied for intervention and the cell activity was detected by SRB assay. As shown in Fig. 1, the activity of HT-29 was less obvious than HT-29/L-OHP after the intervention $(\mathrm{P}<0.05)$, suggesting a stronger resistance of HT-29/L-OHP against L-OHP, and $0.5 \mu \mathrm{g} / \mathrm{ml} \mathrm{L-OHP}$ was selected for further studies.

The expressions of drug-resistant proteins in HT-29 and HT-29/L-OHP cells. Western blot analysis was applied to examine the expressions of drug-resistant proteins in HT-29 and HT-29/L-OHP cells, including MRP-1, P-gp, LOXL2, Survivin, Livin, Bcl-2 and Bax proteins. The result showed higher expressions of MRP-1, P-gp, LOXL2, Survivin, Livin and Bcl-2 but lower expression of Bax in HT-29 than in HT-29/L-OHP (P<0.01) (Fig. 2), which suggested the expression difference of drug-resistant genes between the two cell lines may contribute to the stronger tolerance of HT-29/L-OHP than HT-29 towards L-OHP. Therefore, HT-29/L-OHP was selected to assess the impacts of salidroside in the following experiments.

The effect of salidroside on the cell activity of HT-29/L-OHP and HT-29 cells. The molecular formula of salidroside was shown as Fig. 3A. To explore the effect of salidroside on the cell activity of HT-29/L-OHP cells, different dosages of salidroside were imposed on HT-29 and HT-29/L-OHP cells. Results showed that either of increasing dosage and longer application of salidroside decreased cell activity in HT-29/L-OHP and HT-29 cells (Fig. 3B and C), based on which the accurate dosage and treatment period ( $48 \mathrm{~h}$ ) of salidroside were determined. The cell activity decreased further with the combined use of salidroside and L-OHP (Fig. 3D).

The effect of salidroside on the cell cycle of HT-29/L-OHP and HT-29 cells. In this study, concentration of $80 \mu \mathrm{g} / \mathrm{ml}$ salidroside with $48 \mathrm{~h}$ treatment was applied to evaluate the effect of salidroside on the cell cycle of HT-29/L-OHP and HT-29 cells. The result of FCM was shown in Fig. 4A. Compared with control group, the intervention resulted in more HT-29/L-OHP and HT-29 cells arrested in $\mathrm{G}_{0} / \mathrm{G}_{1}$ stage, less in $\mathrm{G}_{2} / \mathrm{M}$ stage $(\mathrm{P}<0.01)$, whereas almost the same proportion in $\mathrm{S}$ stage (Fig. 4B), suggesting salidroside could inhibit the proliferation of HT-29/L-OHP and HT-29 cells. 
A<smiles>OCC1OC2OC1C(OCCc1ccc(O)cc1)C(O)C2O</smiles>

B

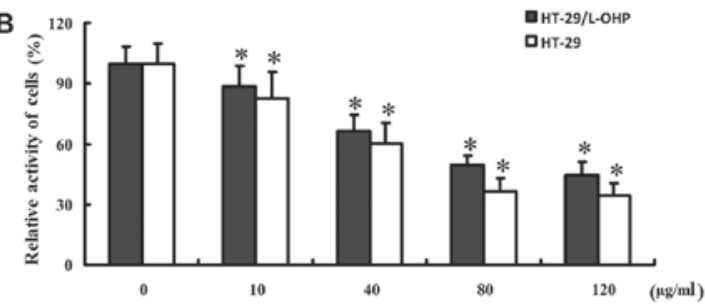

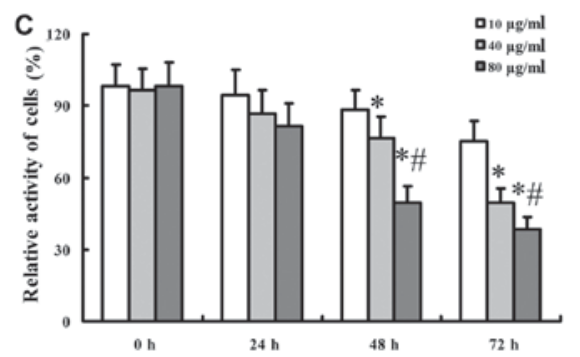

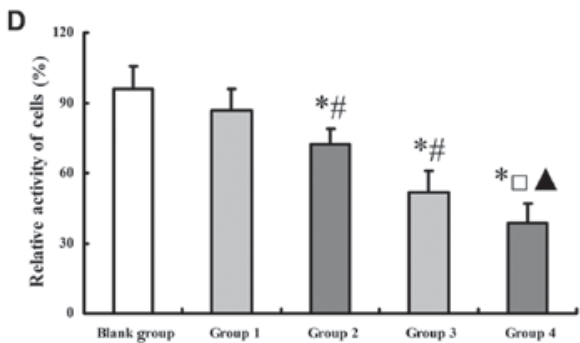

Figure 3. Effect of salidroside on the cell activity of HT-29/L-OHP and HT-29 cells. Image of salidroside molecular structure was shown as (A). Effect of salidroside with different concentrations was illustrated as (B). Results of (C) suggested that salidroside could inhibit the activity of HT-29/L-OHP cells with dose- and time-dependent manner, and combination of salidroside and L-OHP could inhibit activity of HT-29/L-OHP cells more significantly, which was illus-

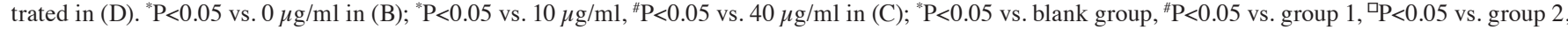
and $\triangle \mathrm{P}<0.05$ vs. group 3 in (D). Group 1 (salidroside $10 \mu \mathrm{g} / \mathrm{ml}$ ); group 2 (salidroside $10 \mu \mathrm{g} / \mathrm{ml}$ combined with L-OHP); group 3 (salidroside $80 \mu \mathrm{g} / \mathrm{ml}$ ); and group 4 (salidroside $80 \mu \mathrm{g} / \mathrm{ml}$ combined with L-OHP).

A

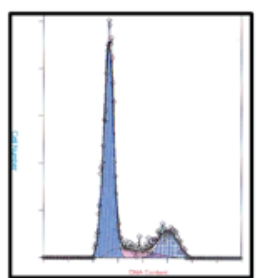

Salidroside group

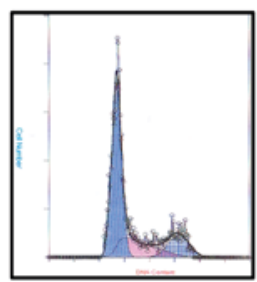

Control group

HT-29/L-OHP cells

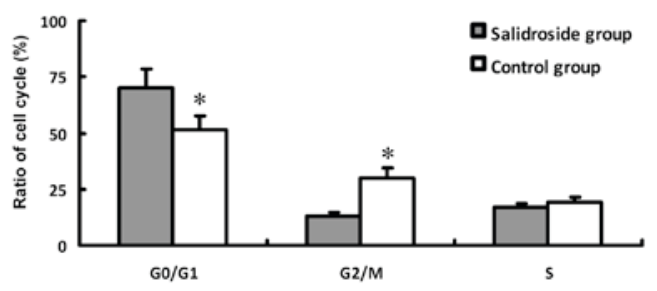

B

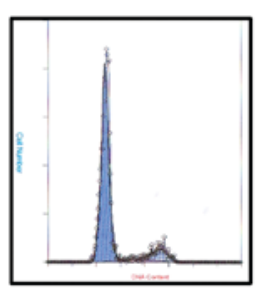

Salidroside group
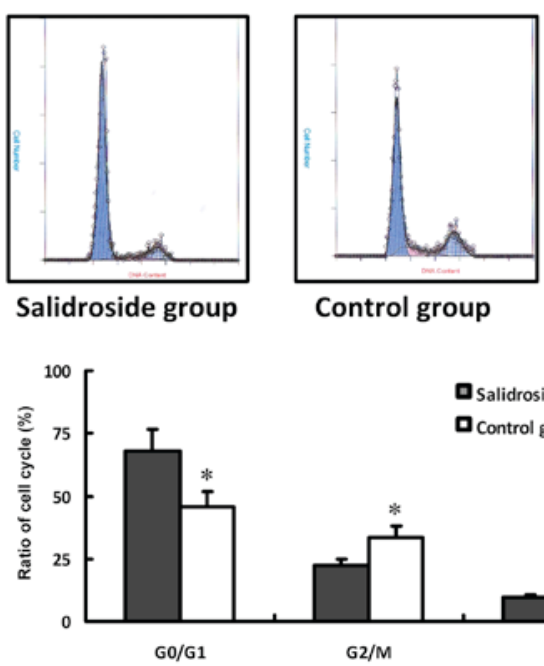

Q Salidroside group
- Control group

HT-29 cells

Figure 4. Cell cycle of HT-29/L-OHP and HT-29 cells in different groups. HT-29/L-OHP and HT-29 cells treated with salidroside respectively showed variation of cell cycle, which was detected by FCM. Compared with control group, the intervention resulted in more HT-29/L-OHP or HT-29 cells arrested in $\mathrm{G}_{0} / \mathrm{G}_{1}$ stage, $(\mathrm{P}<0.01)$, and results of HT-29/L-OHP were shown in (A), results of HT-29 in (B). ${ }^{*} \mathrm{P}<0.01$ vs. control group.

The effect of salidroside on apoptosis of HT-29/L-OHP and HT-29 cells. The effects of salidroside on the apoptosis of HT-29/L-OHP and HT-29 cells were detected under the same experimental dosage and time. The result of FCM was shown in Fig. 5A. In comparison with the control group, the apoptosis rate was higher in HT-29/L-OHP and HT-29 cells after salidroside intervention $(\mathrm{P}<0.01)$ (Fig. 5B), suggesting that salidroside could promote the apoptosis of HT-29/L-OHP and HT-29 cells.

The effect of salidroside on the invasion and migration of HT-29/L-OHP and HT-29 cells. HT-29/L-OHP and HT-29 cells were pretreated by $80 \mu \mathrm{g} / \mathrm{ml}$ salidroside for $48 \mathrm{~h}$ respectively, and then detected with scratch test to evaluate the cell migration activity and Transwell chamber assay to assess the cell invasion capacity. As shown in Fig. 6, the inhibition ratio and invasion descended in a dose-dependent manner after the intervention, indicating that salidroside could inhibit the invasion and migration of HT-29/L-OHP and HT-29 cells.

The effect of salidroside on the expressions of drug-resistant genes and caspase-3 activity in HT-29/L-OHP and HT-29 cells. To explore the molecular mechanism of salidroside on L-OHP resistance cells, the expressions of drug-resistant genes in HT-29/L-OHP were detected. The results indicated decreased expressions of MRP-1, MDR1/P-gp, LOXL2, Survivin, Livin and Bcl-2 but increased Bax in HT-29/L-OHP cells, compared with Control group $(\mathrm{P}<0.05)$ (Fig. 7A-C), which suggested that salidroside could strengthen the sensitivity of HT-29/L-OHP to chemotherapeutic drugs by regulating the expressions of drug-resistant genes. Our results suggested salidroside could inhibit the expressions of Survivin and Livin, two anti-apoptosis proteins that could affect the function of caspase-3. Results indicated that the activity of caspase-3 in HT-29/L-OHP also increased after the intervention, implying salidroside may exert by suppressing the expressions of Survivin and Livin and enhancing caspase-3 
A

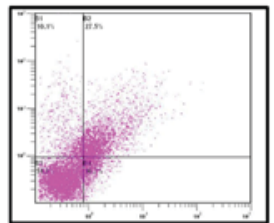

Salidroside group

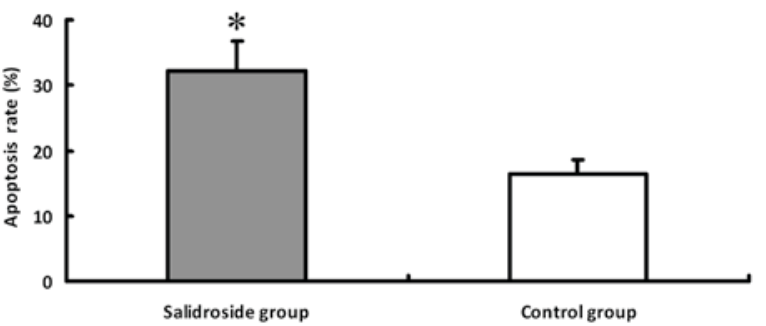

B

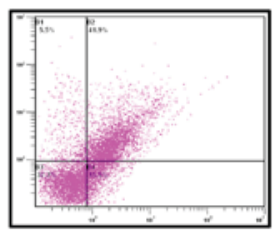

Salidroside group

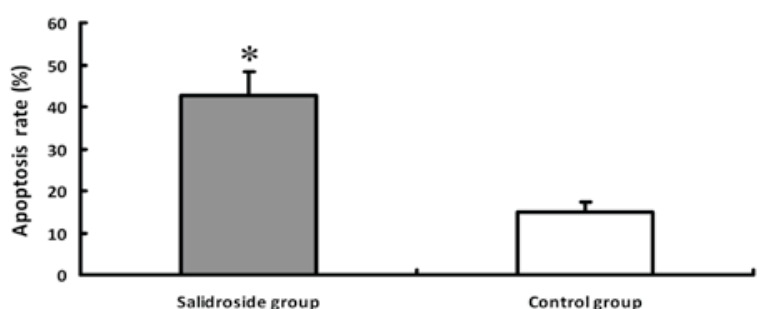

Figure 5. Apoptosis rate of HT-29/L-OHP and HT-29 cells in different groups. HT-29/L-OHP and HT-29 cells treated with salidroside showed variation of apoptosis rate with FCM detection. Compared with control group the apoptosis rate was higher in both cell lines after salidroside intervention $(\mathrm{P}<0.01)$, and results of HT-29/L-OHP were shown in (A), results of HT-29 in $(\mathrm{B}) .{ }^{*} \mathrm{P}<0.01$ vs. control group.

activity (Fig. 7D). HT-29 cells were also treated with salidroside, and then drug-resistant genes and caspase-3 activity were also tested. Results of salidroside on HT-29 cells were similar to those of salidroside on HT-29/L-OHP cells (Fig. 8).

\section{Discussion}

CRC is a common malignant digestive tract tumor in China, which is characterized by the dissatisfactory therapeutic efficacy and poor prognosis $(21,22)$. Chemotherapy plays an important role in the treatment of CRC, which, however, frequently fails and results in recurrence and metastasis due to the drug resistance of CRC cells $(23,24)$. L-OHP, a 3rd generation platinum-based anticancer drug, is the primary component in the first-line chemotherapy for CRC. The cytotoxic and anti-cancer effect of L-OHP is to inhibiting DNA synthesis in the cancer cells by creating crosslink within and between DNA chains $(25,26)$. Although the effect of L-OHP is better than DDP, the resistance of CRC cells against L-OHP decreases the clinical outcomes $(27,28)$. In this study, the cell activity of CRC cells was lower than L-OHP resistance CRC cells after L-OHP intervention, demonstrating the resistance
A

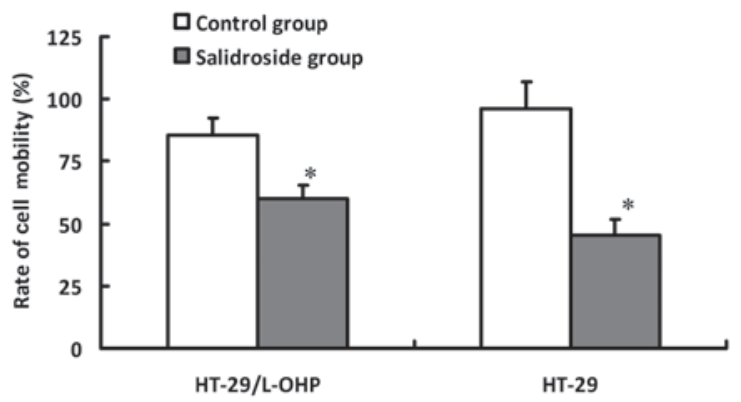

B

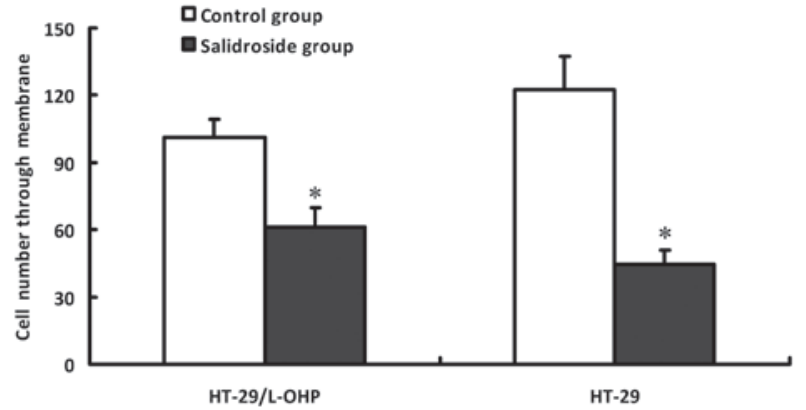

Figure 6. The effects of salidroside on migration and invasion of HT-29/ L-OHP and HT-29 cells. HT-29/L-OHP and HT-29 cells were intervened with salidroside, and then were subjected to scratch assay to detect cell migrating activity (A). Transwell chamber assay to detect cell invasion activity (B). ${ }^{*} \mathrm{P}<0.05$ vs. control group.

of CRC cells to L-OHP (29). Therefore, a combination of innovative drugs and L-OHP may improve the effectiveness of chemotherapy.

Rhodiolae, a traditional medicinal plant, is widely used in Far East and Russia $(15,16)$ for immunity improvement $(30)$, anti-oxidation/cancer $(31,32)$, cardiovascular protection (33), glycol-/lipid-metabolism enhancement and neuron protection $(34,35)$. Salidroside is the active ingredient of Rhodiolae, which has obvious suppression on renal clear cell cancer, lung cancer and breast cancer (17-19). Recent evidence has demonstrated that salidroside could inhibit the proliferation of CRC cells by regulating the JAK2/STAT3 signaling. However, whether Rhodiolae can relieve the resistance of CRCs against L-OHP remains unclear. In this study, we observed higher sensitivity of CRC cells to L-OHP after salidroside intervention, suggesting the positive effect of salidroside on the resistance of CRC cells against L-OHP. The results indicated that salidroside can impede the transition of HT-29/L-OHP and HT-29 cells from $G_{0} / G_{1}$ stage to $G_{2} / M$ stage, whereas it also promotes the apoptosis and inhibits the invasion and migration of HT-29/L-OHP and HT-29 cells. Our results revealed multiple pathways of salidroside's suppression on HT-29/L-OHP cells and suggested the combined use of salidroside in the L-OHP oriented chemotherapy can improve the clinical effect.

Previous studies revealed the participations of agent pump protein, detoxification pathway and DNA repair in the L-OHP resistance (36-39). To explore the mechanism of salidroside on HT-29/L-OHP and HT-29 cells, we compared the expressions of MRP-1, P-gp, LOXL2, Survivin, Livin and Bcl-2 before and after salidroside intervention. MRP-1 and P-gp, located at the membrane of cancer cells, could pump 
A

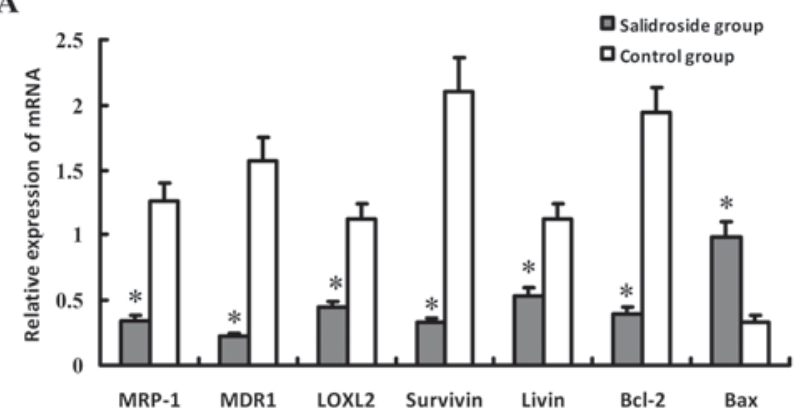

D

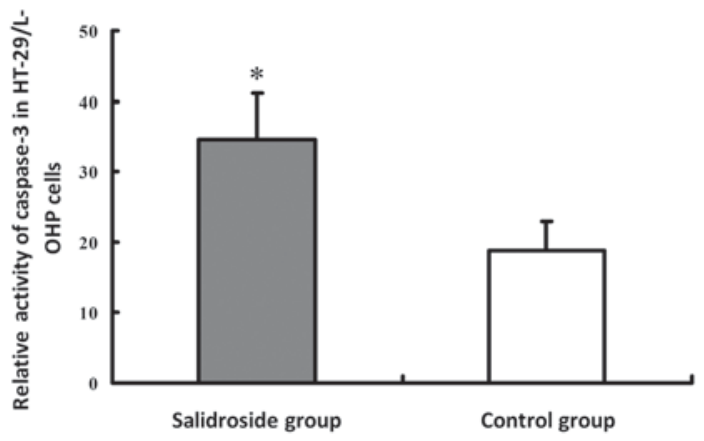

B

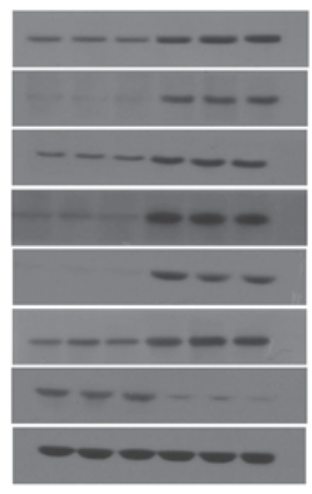

MRP-1

P-gp

LOXL2

Survivin

Livin

Bcl-2

Bax

GAPDH

$\begin{array}{llllll}1 & 1 & 1 & 2 & 2 & 2\end{array}$

Figure 7. Effect of salidroside on the expressions of MRP-1, MDR1/P-gp, LOXL2, Survivin, Livin, Bcl-2, Bax genes and caspase-3 activity in HT-29/L-OHP cells. HT-29/L-OHP cells were treated with salidroside, and then were subjected to real-time quantitative PCR and western blot analyses, to detect the mRNA or protein expression levels of MRP-1, MDR1/P-gp, LOXL2, Survivin, Livin, Bcl-2, Bax genes. Results showed that expressions of MRP-1, MDR1/P-gp, LOXL2, Survivin, Livin and Bcl-2 decreased but Bax increased in HT-29/L-OHP cells, which was illustrated in (A-C). Activity of caspase-3 in HT-29/L-OHP also increased after salidroside intervention, as shown in (D). " $\mathrm{P}<0.05$ vs. control group.

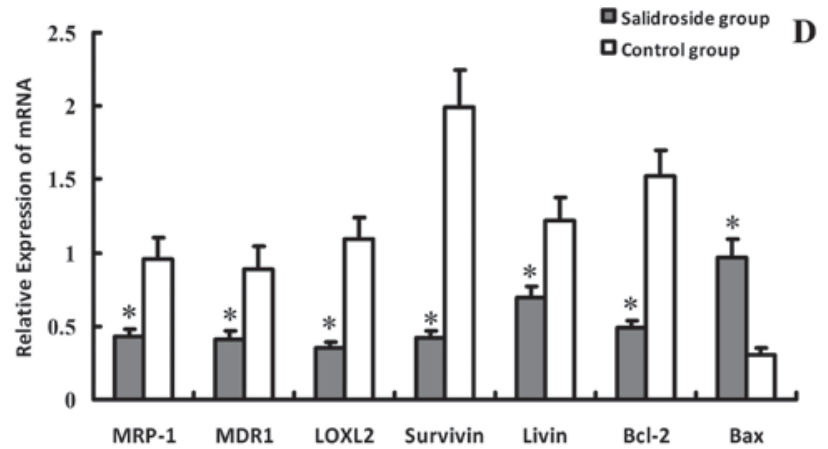

B

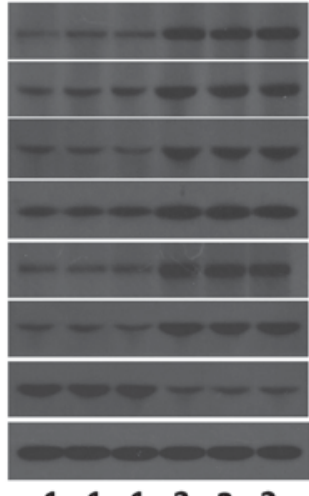

$\begin{array}{llllll}1 & 1 & 1 & 2 & 2 & 2\end{array}$
C
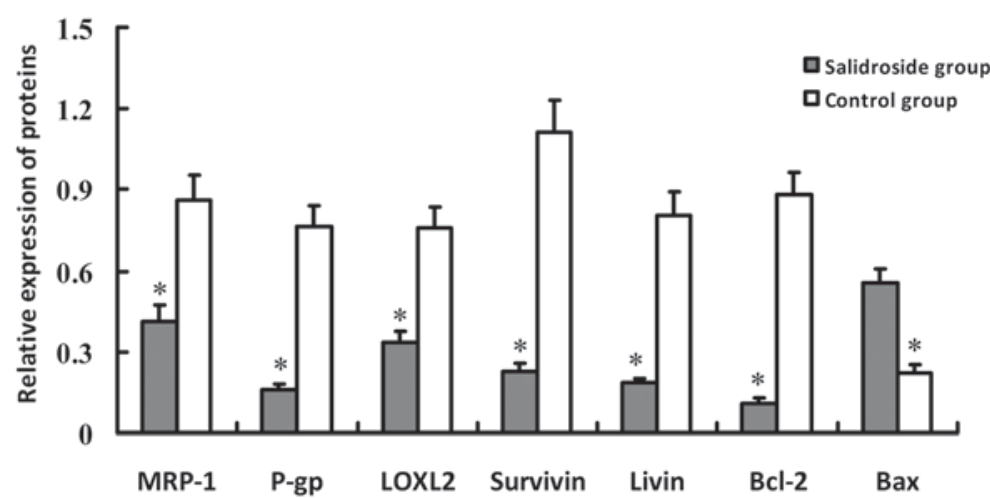
chemotherapeutic drugs out via endoergic reaction $(40,41)$. Recent study has demonstrated that LOXL2 can inhibit the diffusion of gemcitabine in the pancreatic ductal adenocarcinoma (PDAC) (42). Survivin (43), Livin (44) and Bcl-2 could enhance the anti-apoptosis of cancer cells, while in contrast Bax can promote apoptosis. Therefore, the ratio of Bcl-2/Bax in the dimer composed by the two proteins determines the apoptosis capacity of cancer cells $(45,46)$. In this study, we observed declines of MRP-1, MDR1/P-gp, LOXL2, Survivin, Livin and Bcl-2, whereas increasing expression of Bax after salidroside intervention, suggesting salidroside can relieve the resistance of $\mathrm{CRC}$ cells to $\mathrm{L}-\mathrm{OHP}$ by regulating these drug-resistant genes. Results of salidroside on HT-29 cells were similar to those of salidroside on HT-29/L-OHP cells. However, more studies are needed to explore the interaction between specific molecules, and in further study we'll investigate effects of salidroside on other CRC cell lines.

In conclusion, our study revealed that salidroside could decrease the activity and invasion capacity of HT-29/ L-OHP, and treatment of salidroside related to apoptosis of cancer cells by regulating the expressions of related genes, possibly due to inhibiting the expressions of MRP-1, MDR1/P-gp, LOXL2, Survivin, Livin and Bcl-2, whereas promoting the expression of Bax genes. Therefore, we suggest the combined application of salidroside in the L-OHP oriented chemotherapy in order to improve the clinical efficacy for CRC patients. However, further researches are needed to confirm this conclusion via in vivo experiments and large-scale clinical studies.

\section{References}

1. Favoriti P, Carbone G, Greco M, Pirozzi F, Pirozzi RE and Corcione F: Worldwide burden of colorectal cancer: A review. Updates Surg 68: 7-11, 2016.

2. Chen W, Zheng R, Zeng H and Zhang S: The incidence and mortality of major cancers in China, 2012. Chin J Cancer 35: 73, 2016.

3. Bode AM, Dong Z and Wang H: Cancer prevention and control: Alarming challenges in China. Natl Sci Rev 3: 117-127, 2016.

4. Rogowski W and Sulżyc-Bielicka V: Optimal duration of a first-line palliative chemotherapy in disseminated colorectal cancer-a review of the literature from a developing country perspective. Contemp Oncol (Pozn) 20: 210-214, 2016.

5. Zhao L, Liu R, Zhang Z, Li T, Li F, Liu H and Li G: Oxaliplatin/fluorouracil-based adjuvant chemotherapy for locally advanced rectal cancer after neoadjuvant chemoradiotherapy and surgery: A systematic review and meta-analysis of randomized controlled trials. Colorectal Dis 18: 763-772, 2016.

6. Betge J, Barat A, Murphy V, Hielscher T, Van Grieken NC, Belle S, Zhan T, Härtel N, Kripp M, Bacon O, et al: Outcome of colorectal cancer patients treated with combination bevacizumab therapy: A pooled retrospective analysis of three european cohorts from the angiopredict initiative. Digestion 94: 129-137, 2016.

7. Suzuki S, Shimazaki J, Morishita K, Koike N, Harada N, Hayashi T and Suzuki M: Efficacy and safety of oxaliplatin, bevacizumab and oral S-1 for advanced recurrent colorectal cancer. Mol Clin Oncol 5: 391-394, 2016.

8. Zhang X, Chen Y, Hao L, Hou A, Chen X, Li Y, Wang R, Luo P, Ruan Z, Ou J, et al: Macrophages induce resistance to 5-fluorouracil chemotherapy in colorectal cancer through the release of putrescine. Cancer Lett 381: 305-313, 2016.

9. Garborg K: Colorectal cancer screening. Surg Clin North Am 95: 979-989, 2015.

10. De Divitiis C, Nasti G, Montano M, Fisichella R, Iaffaioli RV and Berretta M: Prognostic and predictive response factors in colorectal cancer patients: Between hope and reality. World J Gastroenterol 20: 15049-15059, 2014.
11. Wang W, Liu J, Qi J, Zhang J, Zhu Q, Ma J and Qin C: Downregulation of RLIP76 is associated with vincristine resistance in human colorectal cancer HCT-8/VCR cells. Int J Oncol 49: 1505-1512, 2016.

12. OddoD, SennottEM,BaraultL, ValtortaE, ArenaS, Cassingena A, Filiciotto G, Marzolla G, Elez E, van Geel RM, et al: Molecular landscape of acquired resistance to targeted therapy combinations in BRAF-mutant colorectal cancer. Cancer Res 76: 4504-4515, 2016

13. Wang Z, Zhang L, Ni Z, Sun J, Gao H, Cheng Z, Xu J and Yin P: Resveratrol induces AMPK-dependent MDR1 inhibition in colorectal cancer HCT116/L-OHP cells by preventing activation of NF- $\kappa \mathrm{B}$ signaling and suppressing CAMP-responsive element transcriptional activity. Tumour Biol 36: 9499-9510, 2015.

14. Li F, Tang H, Xiao F, Gong J, Peng Y and Meng X: Protective effect of salidroside from Rhodiolae radix on diabetes-induced oxidative stress in mice. Molecules 16: 9912-9924, 2011.

15. Zheng KY, Guo AJ, Bi CW, Zhu KY, Chan GK, Fu Q, Xu SL, Zhan JY, Lau DT, Dong TT, et al: The extract of Rhodiolae Crenulatae Radix et Rhizoma induces the accumulation of HIF-1 $\alpha$ via blocking the degradation pathway in cultured kidney fibroblasts. Planta Med 77: 894-899, 2011.

16. Li F, Xiao F, Gong J and Yu T: Applied orthogonal experiment design for the optimum microwave-assisted extraction conditions of polysaccharides from Rhodiolae radix. Afr J Tradit Complement Altern Med 10: 179-185, 2013.

17. Lv C, Huang Y, Liu ZX, Yu D and Bai ZM: Salidroside reduces renal cell carcinoma proliferation by inhibiting JAK2/STAT3 signaling. Cancer Biomark 17: 41-47, 2016.

18. Wang J, Li JZ, Lu AX, Zhang KF and Li BJ: Anticancer effect of salidroside on A549 lung cancer cells through inhibition of oxidative stress and phospho-p38 expression. Oncol Lett 7: 1159-1164, 2014.

19. Zhao G, Shi A, Fan Z and Du Y: Salidroside inhibits the growth of human breast cancer in vitro and in vivo. Oncol Rep 33: 2553-2560, 2015

20. Sun KX, Xia HW and Xia RL: Anticancer effect of salidroside on colon cancer through inhibiting JAK2/STAT3 signaling pathway. Int J Clin Exp Pathol 8: 615-621, 2015.

21. Kraus S, Nabiochtchikov I, Shapira S and Arber N: Recent advances in personalized colorectal cancer research. Cancer Lett 347: 15-21, 2014.

22. Berge E, Thompson $\mathrm{C}$ and Messersmith W: Development of novel targeted agents in the treatment of metastatic colorectal cancer. Clin Colorectal Cancer 10: 266-278, 2011.

23. Temraz S, Mukherji D, Alameddine R and Shamseddine A: Methods of overcoming treatment resistance in colorectal cancer. Crit Rev Oncol Hematol 89: 217-230, 2014.

24. Bian Z, Feng Y, Xue Y, Hu Y, Wang Q, Zhou L, Liu Z, Zhang J, Yin Y, Gu B and Huang Z: Down-regulation of SNX1 predicts poor prognosis and contributes to drug resistance in colorectal cancer. Tumour Biol 37: 6619-6625, 2016.

25. Suzuki S and Tanigawara Y: Forced expression of S100A10 reduces sensitivity to oxaliplatin in colorectal cancer cells. Proteome Sci 12: 26, 2014.

26. Toki MI, Saif MW and Syrigos KN: Hypersensitivity reactions associated with oxaliplatin and their clinical management. Expert Opin Drug Saf 13: 1545-1554, 2014.

27. Bi J, Bai Z, Ma X, Song J, Guo Y, Zhao J, Yi X, Han S and Zhang Z: Txr1: An important factor in oxaliplatin resistance in gastric cancer. Med Oncol 31: 807, 2014.

28. Chen CC, Chen LT, Tsou TC, Pan WY, Kuo CC, Liu JF, Yeh SC, Tsai FY, Hsieh HP and Chang JY: Combined modalities of resistance in an oxaliplatin-resistant human gastric cancer cell line with enhanced sensitivity to 5-fluorouracil. Br J Cancer 97: 334-344, 2007.

29. Hu CJ, Wang B, Tang B, Chen BJ, Xiao YF, Qin Y, Yong X, Luo G, Zhang JW, Zhang D, et al: The FOXM1-induced resistance to oxaliplatin is partially mediated by its novel target gene Mcl-1 in gastric cancer cells. Biochim Biophys Acta 1849: 290-299, 2015.

30. Bocharova OA, Serebriakova RV and Bodrova NB: Preventive effect of Rhodiolae rosea in spontaneous liver carcinogenesis in a mice model of high-tumor strain. Vestn Ross Akad Med Nauk 5: 41-43, 1994 (In Russian).

31. Lee OH, Kwon YI, Apostolidis E, Shetty K and Kim YC: Rhodiola-induced inhibition of adipogenesis involves antioxidant enzyme response associated with pentose phosphate pathway. Phytother Res 25: 106-115, 2011. 
32. Bassa LM, Jacobs C, Gregory K, Henchey E, Ser-Dolansky J and Schneider SS: Rhodiola crenulata induces an early estrogenic response and reduces proliferation and tumorsphere formation over time in MCF7 breast cancer cells. Phytomedicine 23: 87-94, 2016.

33. Yu L, Qin Y, Wang Q, Zhang L, Liu Y, Wang T, Huang L, Wu L and Xiong H: The efficacy and safety of Chinese herbal medicine, Rhodiola formulation in treating ischemic heart disease: A systematic review and meta-analysis of randomized controlled trials. Complement Ther Med 22: 814-825, 2014.

34. Wang J, Rong X, Li W, Yang Y, Yamahara J and Li Y: Rhodiola crenulata root ameliorates derangements of glucose and lipid metabolism in a rat model of the metabolic syndrome and type 2 diabetes. J Ethnopharmacol 142: 782-788, 2012.

35. Xiao L, Li H, Zhang J, Yang F, Huang A, Deng J, Liang M, Ma F, Hu M and Huang Z: Salidroside protects Caenorhabditis elegans neurons from polyglutamine-mediated toxicity by reducing oxidative stress. Molecules 19: 7757-7769, 2014.

36. Helmbach H, Kern MA, Rossmann E, Renz K, Kissel C, Gschwendt B and Schadendorf D: Drug resistance towards etoposide and cisplatin in human melanoma cells is associated with drug-dependent apoptosis deficiency. J Invest Dermatol 118: 923-932, 2002.

37. Hunakova L, Gronesova P, Horvathova E, Chalupa I, Cholujova D, Duraj J and Sedlak J: Modulation of cisplatin sensitivity in human ovarian carcinoma A2780 and SKOV3 cell lines by sulforaphane. Toxicol Lett 230: 479-486, 2014.

38. Murphy RF, Komlodi-Pasztor E, Robey R, Balis FM, Farrell NP and Fojo T: Retained platinum uptake and indifference to p53 status make novel transplatinum agents active in platinum-resistant cells compared to cisplatin and oxaliplatin. Cell Cycle 11: 963-973, 2012

39. Gourdier I, Del Rio M, Crabbé L, Candeil L, Copois V, Ychou M, Auffray C, Martineau P, Mechti N, Pommier Y and Pau B: Drug specific resistance to oxaliplatin is associated with apoptosis defect in a cellular model of colon carcinoma. FEBS Lett 529: 232-236, 2002
40. Sui H, Fan ZZ and Li Q: Signal transduction pathways and transcriptional mechanisms of ABCB1/Pgp-mediated multiple drug resistance in human cancer cells. J Int Med Res 40: 426-435, 2012.

41. Wang Y, Liu L, Liu X, Zhang H, Liu J, Feng B, Shang Y, Zhou L, Wu K, Nie Y, et al: Shugoshin1 enhances multidrug resistance of gastric cancer cells by regulating MRP1, Bcl-2 and Bax genes. Tumour Biol 34: 2205-2214, 2013.

42. Le Calvé B, Griveau A, Vindrieux D, Maréchal R, Wiel C, Svrcek M, Gout J, Azzi L, Payen L, Cros J, et al: Lysyl oxidase family activity promotes resistance of pancreatic ductal adenocarcinoma to chemotherapy by limiting the intratumoral anticancer drug distribution. Oncotarget 7: 32100-32112, 2016.

43. Véquaud E, Desplanques G, Jézéquel P, Juin P and Barillé-Nion S: Survivin contributes to DNA repair by homologous recombination in breast cancer cells. Breast Cancer Res Treat 155: 53-63, 2016.

44. Hsieh CH, Lin YJ, Wu CP, Lee HT, Shyu WC and Wang CC: Livin contributes to tumor hypoxia-induced resistance to cytotoxic therapies in glioblastoma multiforme. Clin Cancer Res 21: 460-470, 2015 .

45. Golestani Eimani B, Sanati MH, Houshmand M, Ataei M, Akbarian F and Shakhssalim N: Expression and prognostic significance of bcl-2 and bax in the progression and clinical outcome of transitional bladder cell carcinoma. Cell J 15: 356-363, 2014.

46. Wu S, Liu B, Zhang Q, Liu J, Zhou W, Wang C, Li M, Bao S and Zhu R: Dihydromyricetin reduced Bcl-2 expression via p53 in Human Hepatoma HepG2 Cells. PLoS One 8: e76886, 2013.

This work is licensed under a Creative Commons Attribution-NonCommercial-NoDerivatives 4.0 International (CC BY-NC-ND 4.0) License. 